Comment. Math. Helv. 74 (1999) 345-363

(C) 1999 Birkhäuser Verlag, Basel

0010-2571/99/030345-19\$1.50+0.20/0

Commentarii Mathematici Helvetici

\title{
Controlled Geometry via Smoothing
}

\author{
Peter Petersen ${ }^{\dagger}$, Guofang Wei ${ }^{\ddagger}$ and Rugang $\mathrm{Ye}^{\S}$
}

\begin{abstract}
We prove that Riemannian metrics with a uniform weak norm can be smoothed to having arbitrarily high regularity. This generalizes all previous smoothing results. As a consequence we obtain a generalization of Gromov's almost flat manifold theorem. A uniform Betti number estimate is also obtained.
\end{abstract}

Mathematics Subject Classification (1991). 53C20.

Keywords. Smoothing, regularity of metric, curvature bounds.

\section{Introduction}

An ultimate goal in geometry is to achieve a classification scheme, using natural geometric quantities to characterize the topological type or diffeomorphism type of Riemannian manifolds. While this grand scheme seems to be an impossible dream, its basic philosophy has been a driving force in many important developments in Riemannian geometry. The sphere theorems and various topological finiteness theorems are typical examples. These results are concerned with control of global topology of manifolds, and a crucial point therein is to control, uniformly, the local topology.

Control of local topology often follows from control of local geometry. Here, by local geometry, we mean the local behavior of the metric tensor. On the other hand, control of local geometry is frequently also the essential ingredient for control of global geometry, such as in Cheeger-Gromov's compactness theorem and its various extensions, which can be named geometric finiteness theorems. Notice that some rudimental topological finiteness results are direct corollaries of geometric finiteness theorems. But the significance of the latter goes beyond this. In any case, control of local geometry is obviously a key topic. An interesting and important aspect of this topic is various degrees of control of local geometry needed

\footnotetext{
$\dagger$ Partially supported by NSF and NYI grants.

¥Partially supported by NSF Grant \# DMS9409166.

§Partially supported by NSF Grant \# DMS9401106.
} 
or available in different situations. In $[\mathrm{P}]$, the first author introduced a sequence of norms which provide a certain quantitative measure for local geometric control. These norms can be defined either in terms of the $C^{k, \alpha}$-norms or the $L^{k, p}$-norms for functions, and they are defined on a given scale. For example, the $C^{k, \alpha}$-norm of a Riemannian manifold on scale $r$ is bounded, if it is covered by coordinate charts of size comparable to $r$ such that the metric tensor expressed in the coordinates is uniformly bounded in $C^{k, \alpha}$-norm, and that the coordinate transition functions are uniformly bounded in $C^{k+1, \alpha}$-norm. Note that the local topology is uniformly trivial if one of these norms on some scale is bounded. To admit richer topological and geometric structures under norm bounds, we shall introduce a weak version of these norms. The essential new feature is that we allow coordinate maps to have double points. In spirit, this is similar to replacing an injectivity radius bound by a conjugate radius bound. (Of course, e.g. a weak (harmonic) $C^{0, \alpha}$ bound is so weak that it is far from implying a conjugate radius bound.)

Indeed, our basic theme is to try to find the minimal degree of control of local geometry under which interesting geometric and topological consequences can be drawn. Traditional geometric conditions such as curvature bounds imply various degrees of local geometric control, so we can think from their perspectives. Historically, sectional curvature bounds were the first to be systematically studied. They can roughly be compared with weak $C^{2}$-norm bounds, at least the latter imply the former. Since understanding of sectional curvature bounds has been reached on a good level, it is natural to try to find the minimal degree of local geometric control under which a metric can be approximated by metrics with sectional curvature bounds or weak $C^{2}$-norm bounds (or better bounds). In this paper, we present a result towards this goal, along with some applications. The local geometric control we need is as weak as a bound on the weak harmonic $C^{0, \alpha_{-}}$ norm, or a bound on the weak $L^{1, p}$-norm. These do appear to be the sought-after minimal degree of local geometric control in our set-up.

To formulate the result precisely, we introduce the following classes of Riemannian manifolds. (The definition of the weak norms are given in $\S 2$.)

Definition 1. Given $n \geq 2,0<\alpha<1, p>n$ and function $Q:(0, \infty) \rightarrow[0, \infty)$ which is nondecreasing in $r$ and satisfies $\lim _{r \rightarrow 0} Q(r)=0$, we define

$$
\mathcal{M}(n, \alpha, Q)=\left\{(M, g) \mid \begin{array}{r}
(M, g) \text { is a complete Riemannian manifold, } \operatorname{dim} M=n, \\
\text { the weak harmonic } C^{0, \alpha} \text { norm }\|(M, g)\|_{C^{0, \alpha}, r}^{W, h} \leq Q(r) \\
\text { for all positive } r \leq 1
\end{array}\right\},
$$

and

$$
\mathcal{M}(n, p, Q)=\left\{(M, g) \mid \begin{array}{r}
(M, g) \text { is a complete Riemannian manifold, } \operatorname{dim} M=n, \\
\text { the weak } L^{1, p} \text { norm }\|(M, g)\|_{L^{1, p}, r}^{W} \leq Q(r) \\
\text { for all positive } r \leq 1
\end{array}\right\} .
$$


Remark 1. A priori these two classes seem to be independent. We will prove however that (weak) $L^{1, p}$ bounds actually imply (weak) harmonic $C^{0, \alpha}$ bounds, see Theorem 1.5. A further question is whether (weak) $C^{0, \alpha}$ bounds imply (weak) harmonic $C^{0, \alpha}$ bounds. It is still unanswered.

Remark 2. By Anderson-Cheeger's work $[\mathrm{AC}]$ manifolds with a lower bound for Ricci curvature and a positive lower bound for conjugate radius belong to these two classes.

Theorem 1.1. For every manifold $(M, g)$ in $\mathcal{M}(n, \alpha, Q)$, every $r \in(0,1]$ and every positive number $\epsilon$, there is a metric $g_{\epsilon}$ on $M$ such that

$$
\begin{aligned}
e^{-\epsilon} g & \leq g_{\epsilon} \leq e^{\epsilon} g \\
\left\|\left(M, g_{\epsilon}\right)\right\|_{C^{0, \alpha}, r}^{W} & \leq 2 Q(r), \\
\left\|\left(M, g_{\epsilon}\right)\right\|_{C^{k, \alpha}, r}^{W} & \leq \widetilde{Q}
\end{aligned}
$$

where $k$ is an arbitrary positive integer and $\widetilde{Q}=\widetilde{Q}(n, k, \epsilon, \alpha, Q(r))$ denotes a positive number depending only on $n, k, \epsilon, \alpha$ and $Q(r)$.

Theorem 1.2. For every manifold $(M, g)$ in $\mathcal{M}(n, p, Q)$, every $r \in(0,1]$ and every positive number $\epsilon$, there is a metric $g_{\epsilon}$ on $M$ such that

$$
\begin{aligned}
e^{-\epsilon} g \leq g_{\epsilon} & \leq e^{\epsilon} g \\
\left\|\left(M, g_{\epsilon}\right)\right\|_{L^{1, p}, r}^{W} & \leq 2 Q(r), \\
\left\|\left(M, g_{\epsilon}\right)\right\|_{L^{k, p}, r}^{W} & \leq \widetilde{Q}
\end{aligned}
$$

where $k$ is an arbitrary positive integer and $\widetilde{Q}=\widetilde{Q}(n, k, \epsilon, p, Q(r))$ denotes a positive number depending only on $n, k, \epsilon, p$ and $Q(r)$.

Thus a metric with some regularity (given by the weak norm) can be deformed or smoothed to a nearby one with arbitrarily high regularity. In particular, manifolds with a lower bound on Ricci curvature and a positive lower bound on conjugate radius can be smoothed. Previous smoothing results have been concerned with metrics with various curvature bounds, and involved two independent techniques: the embedding method and the Ricci flow. The embedding technique in smoothing as used by Cheeger-Gromov [CG] consists of embedding (or immersing) a given manifold into a Euclidean space and then perturbing it suitably by a smoothing operation, which is based on the classical convolution process. The smoothing result in [CG] is that metrics on closed manifolds with lower and upper bounds on sectional curvatures and a positive lower bound on injectivity radius can be smoothed to metrics with bounds on all derivatives of the Riemann curvature tensor. Later, by embedding into a Hilbert space instead of a finite dimensional 
space, Abresch $[\mathrm{A}]$ was able to remove the condition on injectivity radius and extend to complete manifolds. More recently Shen $[\mathrm{SH}]$ showed that manifolds with a lower bound on sectional curvatures and a positive lower bound on injectivity radius can be smoothed to having two-sided sectional curvature bounds. The technique of Ricci flow is based on the fundamental work of Hamilton $[\mathrm{H}]$. Using this technique, Bemelmans-Min Oo-Ruh [BMR] obtained the same result as in $[\mathrm{CG}]$ without injectivity radius lower bound, and Shi $[\mathrm{S}]$ obtained the same result as in [A]. Later work considers metrics with other kinds of curvature bound. For example, in [Ga2, Y1, Y2] Gao and Yang dealt with integral bounds on sectional curvatures. In [DWY], Ricci curvature bounds were treated.

By virtue of the available constructions of controlled harmonic coordinates under various curvature bounds, all these smoothing results are consequences of Theorem 1.1 or Theorem 1.2.

As typical applications we present the following two results.

Theorem 1.3. (Betti number estimate) For the class of manifolds $M^{n}$ in $\mathcal{M}(n, \alpha, Q)$, and satisfying $\operatorname{diam}_{M} \leq D$, we have the estimate for the Betti numbers

$$
\sum_{i} b^{i}\left(M^{n}\right) \leq C(n, D, \alpha, Q)
$$

and the estimate for the number of isomorphism classes of rational homotopy groups

$$
\pi_{q}(M) \otimes \mathbb{Q} \leq C(n, q, D, \alpha, Q) \text { for } q \geq 2 .
$$

(1.7) follows from Theorem 1.1 and Gromov's uniform betti number estimate regarding sectional curvature [G2]. This estimate can also be proved directly using Toponogov type comparison estimate introduced in $[\mathrm{W}]$, see $[\mathrm{PW}]$ for details. In [W] the same estimate (1.7) is given for the class of manifolds satisfying $\operatorname{Ric}_{M} \geq$ $-(n-1) H$, conj $\geq r_{0}$ and $\operatorname{diam}_{M} \leq D$. (1.8) follows from Theorem 1.1, 1.2 and the result in [R, Theorem 0.3].

Theorem 1.4. There exists an $\epsilon=\epsilon(n, \alpha, Q)>0$ such that if a manifold $M^{n}$ belongs to $\mathcal{M}(n, \alpha, Q)$ and diam $\leq \epsilon$, then $M$ is diffeomorphic to an infranilmanifold.

This generalizes Gromov's almost flat manifold theorem [G1] as well as its generalization in [DWY]. (The proof is simple: combine Theorem 1.1 with [G1].)

Remark. By Theorem 1.2, Theorem 1.3 and 1.4 also hold if we replace the class $\mathcal{M}(n, \alpha, Q)$ by $\mathcal{M}(n, p, Q)$. (The constants will depend on $p$ instead of $\alpha$.)

By the Sobolev embedding, $L^{1, p}$ harmonic norm controls $C^{0, \alpha}$ harmonic norm. The following result demonstrates the relation between $L^{1, p}$-norm and $C^{0, \alpha}$ harmonic norm. 
Theorem 1.5. Let $Q(r)$ be a function as in Definition 1 and $p>n$. Then for any Riemannian manifold $M^{n}$ with $\|(M, g)\|_{L^{1, p}, r}\left(\|(M, g)\|_{L^{1, p}, r}^{W}\right) \leq Q(r)$, there holds

$$
\|(M, g)\|_{L^{1, p}, r}^{h}\left(\|(M, g)\|_{L^{1, p}, r}^{W, h}\right) \leq C(n, p) Q(r),
$$

where $C(n, p)$ is a constant depending only on $n, p$. In other words, controlled harmonic coordinates exist on $M$ (in case of weak norm, the coordinates are allowed to have double points).

On account of this result, Theorem 1.2 follows from Theorem 1.1.

The proof of Theorem 1.1 uses the embedding method in [A]. Roughly speaking, we embed a given Riemannian manifold into the Hilbert space of $L^{2}$-functions on it, and then use the embedding to pull back the $L^{2}$-metric of the Hilbert space. The crucial point is of course to find a suitable embedding, such that the pullback metric will enjoy nice properties. In $[\mathrm{A}]$, the embedding is defined in terms of distance functions. In our situation, these functions are not appropriate, and we employ instead solutions of a canonical geometric partial differential equation. Now if e.g. the harmonic $C^{0, \alpha}$-norm of the manifold is bounded, then a uniform pointwise bound on sectional curvatures will hold for the pull-back metric, and hence we can apply the smoothing results for metrics with sectional curvature bounds as given e.g. in $[\mathrm{A}]$ or $[\mathrm{S}]$.

If we only assume that the weak harmonic $C^{0, \alpha}$-norm of the manifold is bounded, i.e. it is in the class $\mathcal{M}(n, \alpha, Q)$, the global embedding is generally not under control. To remedy the situation, we follow the idea in $[\mathrm{A}]$ of employing instead local embeddings. In $[\mathrm{A}]$, Abresch uses the exponential map to lift local patches of the manifold and his local embeddings are exactly embeddings of these lifted patches. In our situation, the exponential map is not suitable. Our substitute for it is the coordinate maps. Thus we use them to lift local patches, and construct embeddings of the lifted patches via the same geometric partial differential equation as mentioned before. To make sure that the pull-back metrics induced by these local embeddings descend to the local patches and that the resulting metrics patch together to define a metric globally, it is crucial to require the embeddings to be equivariant under isometries. Since our embeddings are defined in terms of solutions of a canonical geometric PDE, they naturally share this equivariance property.

The above scheme (suitably modified) can also be applied to give an alternative proof of Theorem 1.2. This is of course more involved than deriving Theorem 1.2 from Theorem 1.1 and 1.5. However, more geometric structures can be seen from this alternative approach. As before, a given Riemannian manifold (in the class $\mathcal{M}(n, p, Q))$ will be embedded into the Hilbert spaces of $L^{2}$-functions on it, and the pull-back metrics descend to yield a new metric on the underlying manifold. But these metrics satisfy here an integral bound on sectional curvatures rather than a pointwise bound. This is a new situation. To handle it, we can apply the Ricci flow and follow the arguments in [DWY]. The details will appear elsewhere. 


\section{Norm and Weak Norm}

Definition 2. Fix an integer $k \geq 0$ and a number $0 \leq \alpha \leq 1$. The $C^{k, \alpha}$-norm of an $n$-dimensional Riemannian manifold $(M, g)$ on scale $r>0,\|(M, g)\|_{C^{k, \alpha}, r}$, is defined to be the infimum of positive numbers $Q$ such that there exist embeddings:

$$
\varphi_{\tau}: \mathbb{B}(0, r) \subset \mathbb{R}^{n} \rightarrow U_{\tau} \subset M
$$

$(\mathbb{B}(0, r)$ denotes the closed Euclidean ball of radius $r$ centered at the origin) with images $U_{\tau}, \tau \in \mathcal{I}$ (an index set), with the following properties:

1) $e^{-2 Q} \delta_{i j} \leq g_{\tau, i j} \leq e^{2 Q} \delta_{i j}$

2) Every metric ball $B\left(p, \frac{r}{10} e^{-Q}\right), p \in M$ lies in some set $U_{\tau}$, 3) $r^{|l|+\alpha}\left\|\partial^{l} g_{\tau, i j}\right\|_{C^{\alpha}} \leq Q$ for all multi-indices $l$ with $0 \leq|l| \leq k$.

Here $g_{\tau, i j}$ denote the coefficients of $g_{\tau}=\varphi_{\tau}^{*} g$ on $\mathbb{B}(0, r)$, and $\delta_{i j}$ are the Kronecker symbols.

Remark. Note that this definition is slightly different from the corresponding one in $[\mathrm{P}]$, where in addition the (rescaled) $C^{k+1, \alpha}$-norm of the transition functions are required to be under control. For convenience, we can call the $C^{k, \alpha}$-norm (of Riemannian manifolds) as defined in $[\mathrm{P}]$ the strong $C^{k, \alpha}$-norm. (Note however that the "strong" harmonic $C^{k, \alpha}$-norm is equivalent to the harmonic $C^{k, \alpha}$-norm.)

We define the harmonic $C^{k, \alpha}$-norm on scale $r,\|(M, g)\|_{C^{k, \alpha}, r}^{h}$, by requiring additionally the following

4) $\varphi_{\tau}^{-1}: U_{\tau} \rightarrow \mathbb{R}^{n}$ is harmonic,

which is equivalent to saying that

$\left.4^{\prime}\right)$ id $: \mathbb{B}(0, r) \rightarrow \mathbb{B}(0, r)$ is harmonic with respect to $g_{\tau}$ on the domain and the Euclidean metric on the target, which is in turn equivalent to saying that

$$
\sum_{i} \partial_{i}\left(g_{\tau}^{i j} \sqrt{\operatorname{det} g_{\tau, i j}}\right)=0
$$

for all $j$.

If $k \geq 1$ and $p>n$ (when $k=1$ ) or $p>\frac{n}{2}$ (when $k \geq 2$ ), then we define the $L^{k, p}$-norm on the scale of $r,\|(M, g)\|_{L^{k, p}, r}$, by retaining 1$)$ and 2$)$, and replacing 3) by

$\left.3^{\prime}\right) r^{|l|-\frac{n}{p}}\left\|\partial^{l} g_{\tau, i j}\right\|_{L^{p}} \leq Q$ for all $1 \leq|l| \leq k$.

The harmonic $L^{k, p}$-norm is defined similarly.

For any choice of these norms, it is clear that the local topology is trivial on some uniform scale for any class of manifolds with uniformly bounded norm. (Note that the injectivity radius may not be uniformly positive though.) To allow nontrivial local topology, we introduce the weak norms \|\|$_{C^{k, \alpha}, r}^{W}$ and \|\|$_{L^{k, p}, r}^{W}$, which are defined in identical ways except that each $\varphi_{\tau}: \mathbb{B}(0, r) \rightarrow U_{\tau}$ is assumed to be a local diffeomorphism instead of diffeomorphism. The corresponding weak harmonic norms \|\|$_{C^{k, \alpha}, r}^{W, h}$ and \|\|$_{L^{k, p}, r}^{W, h}$ are defined in a similar way, with 4) being replaced by $\left.4^{\prime}\right)$. 
Note that (weak) harmonic norms dominate (weak) norms on the same scale. We also have \|\|$_{, r}^{W} \leq\|\|_{, r}$ and \|\|$_{, r}^{W, h} \leq\|\|_{r}^{h}$. All norms are continuous and non-decreasing in $r$. If $(M, g)$ is sufficiently smooth, these norms converge to zero as $r \rightarrow 0$. Furthermore, (weak) $C^{k, \alpha}\left(L^{k, p}\right)$ norms vary continuously in the $C^{k, \alpha}\left(L^{k, p}\right)$ topology of Riemannian manifolds. See $[\mathrm{P}]$ for the relevant details.

We point out that $\mathbb{R}^{n}$ is the only space with norm $=0$ on all scales. And flat manifolds are the only spaces with weak norm $=0$ on all scales.

Conventional geometric conditions such as curvature bounds imply norm bounds. Such implications are mostly contained in constructions of controlled harmonic coordinates and are a crucial ingredient for various compactness theorems. To have a clear perspective, we collect these results in the following proposition.

Proposition 2.1. There are positive functions $Q(H, \rho, r, p)$ and $Q_{1}(H, r, p)$ of $H \geq 0, \rho>0, r>0, p>n$ and of $H \geq 0, r>0, p>n$ respectively, satisfying $\lim _{r \rightarrow 0} Q(H, \rho, r, p)=0, \lim _{r \rightarrow 0} Q_{1}(H, r, p)=0$ such that for manifolds $M^{n}$ with a) $|K| \leq H$, inj $\geq \rho$, then $\|(M, g)\|_{L^{2, p}, r}^{h} \leq Q(H, \rho, r, p)$;

b) $|K| \leq H$, then $\|(M, g)\|_{L^{2, p}, r}^{W, h} \leq Q_{1}(H, r, p)$;

c) $|\mathrm{Ric}| \leq(n-1) H$, inj $\geq \rho$, then $\|(M, g)\|_{L^{2, p}, r}^{h} \leq Q(H, \rho, r, p)$;

d) Ric $\geq-(n-1) H$, inj $\geq \rho$, then $\|(M, g)\|_{L^{1, p}, r}^{h} \leq Q(H, \rho, r, p)$;

e) Ric $\geq-(n-1) H$, conj $\geq \rho$, then $\|(M, g)\|_{L^{1, p}, r}^{W, r} \leq Q(H, \rho, r, p)$.

These results follow from works of Jost-Karcher [JK], Anderson [AN] and Anderson-Cheeger $[\mathrm{AC}]$. (See also Gao [Ga1, Ga2] for results along these lines.)

We now prove Theorem 1.5.

Proof of Theorem 1.5. We will prove the case when $\|(M, g)\|_{L^{1, p}, r} \leq Q(r)$. The other case is exactly the same. By definition of $L^{1, p}$ norm, we have a collection of diffeomorphisms

$$
\varphi_{\tau}: \mathbb{B}(0, r) \rightarrow U_{\tau} \subset M
$$

satisfying

$$
r^{1-\frac{n}{p}}\left\|\partial g_{\tau, i j}\right\|_{L^{p}, \mathbb{B}(0, r)} \leq Q
$$

Note that the norm is scale invariant, namely

$$
\left\|\left(M, r^{-2} g\right)\right\|_{L^{1, p}, 1}=\|(M, g)\|_{L^{1, p}, r} \leq Q(r) .
$$

So with the scaled pull back metric $r^{-2} \varphi_{\tau}^{*} g$, we have

$$
\left\|\partial r^{-2} g_{\tau, i j}\right\|_{L^{p}, \mathbb{B}(0,1)} \leq Q(r) .
$$

On the Euclidean ball $\mathbb{B}(0,1)$ equipped with the metric $r^{-2} \varphi_{\tau}^{*} g$, solve the Dirichlet boundary value problem:

$$
\psi_{\tau}:\left(\mathbb{B}(0,1), r^{-2} \varphi_{\tau}^{*} g\right) \rightarrow \mathbb{R}^{n}, \text { with } \Delta \psi_{\tau}=0 \text { and }\left.\psi_{\tau}\right|_{\partial \mathbb{B}(0,1)}=\left.\operatorname{Id}\right|_{\partial \mathbb{B}(0,1)},
$$


where Id is the identity map Id: $\mathbb{B}(0,1) \rightarrow \mathbb{R}^{n}$ with $\mathbb{B}(0,1)$ equipped with the metric $r^{-2} \varphi_{\tau}^{*} g$. We will show that $\psi_{\tau}$ is also a coordinate. Now $\Delta\left(\operatorname{Id}-\psi_{\tau}\right)=\Delta \operatorname{Id}$. By the elliptic estimate for divergence operators (see [P, Appendix A])

$$
\begin{aligned}
\left\|\operatorname{Id}-\psi_{\tau}\right\|_{2, p, \mathbb{B}(0,1)} & \leq C_{1}(n, p)\|\Delta \operatorname{Id}\|_{p, \mathbb{B}(0,1)} \\
& \leq C_{1}(n, p)\left\|\partial r^{-2} g_{\tau, i j}\right\|_{p, \mathbb{B}(0,1)} \leq C_{1}(n, p) Q(r) .
\end{aligned}
$$

Since $\lim _{r \rightarrow 0} Q(r)=0$, fix an $0<\epsilon_{0} \ll 1$, there is an $r_{0}>0$ such that $C_{1}(n, p) Q\left(r_{0}\right) \leq \epsilon_{0}$. Therefore $\psi_{\tau}$ is a harmonic coordinate on the ball $\mathbb{B}(0,1)$ for the metric $r^{-2} \varphi_{\tau}^{*} g$ when $r \leq r_{0}$. Moreover the $L^{1, p}$-norm of the metric components with respect to $\psi_{\tau}$ is bounded by $C(n, p) Q(r)$. By scaling back, we have harmonic coordinate on $\mathbb{B}(0, r)$ with respect to the metric $\psi_{\tau}^{*} g$ and $\|(M, g)\|_{L^{1, p}, r}^{h} \leq C(n, p) Q(r)$.

\section{Smoothing}

As explained in the introduction, our strategy is to first achieve sectional curvature bounds by embedding into the Hilbert space of $L^{2}$-functions. This is done in the next two sections. The higher regularity smoothing then easily follows from known smoothing results.

Consider $(M, g) \in \mathcal{M}(n, \alpha, Q)$. We have a collection of local diffeomorphisms

$$
\varphi_{\tau}: \mathbb{B}(0, r) \rightarrow U_{\tau} \subset M
$$

satisfying 1), 2), 3) and $4^{\prime}$ ) of Definition 2.

In the next section we will construct a canonical embedding

$$
F_{\tau}:\left(\mathbb{B}(0, r), g_{\tau}\right) \rightarrow L^{2}\left(\mathbb{B}(0, r), g_{\tau}\right),
$$

where $g_{\tau}=\varphi_{\tau}^{*} g$. We use $F_{\tau}$ to pullback the $L^{2}$ metric of $L^{2}\left(\mathbb{B}(0, r), g_{\tau}\right)$ to produce a new metric $\tilde{g}_{\tau}$ on $\mathbb{B}(0, r)$. This construction works for general metrics on $\mathbb{B}(0, r)$, and has the following equivariance property, which will be proved in $\S 5$. Namely, if $g_{1}, g_{2}$ are two metrics on $\mathbb{B}(0, r)$ such that there is an isometric embedding

$$
\psi:\left(\mathbb{B}(0, r), g_{1}\right) \rightarrow\left(\mathbb{B}(0, r), g_{2}\right)
$$

and if $\tilde{g}_{1}, \tilde{g}_{2}$ are obtained via the above construction, then

$$
\psi:\left(\mathbb{B}(0, r), \tilde{g}_{1}\right) \rightarrow\left(\mathbb{B}(0, r), \tilde{g}_{2}\right)
$$

is also an isometric embedding. Granted this (see Proposition 4.4) we have

Proposition 3.1. There exists a smooth metric $\bar{g}$ on $M$ such that the pullback of $\bar{g}$ by $\varphi_{\tau}$ is exactly $\tilde{g}_{\tau}$. 
Proof. Let $r_{1}=\frac{r}{10} e^{-Q}$. Then for every $p \in M, B_{g}\left(p, r_{1}\right) \subset U_{\tau}$ for some $\tau$. It follows that there exists a $\tilde{p} \in \mathbb{B}(0, r)$ such that $B_{g_{\tau}}\left(\tilde{p}, r_{1}\right) \subset \mathbb{B}(0, r)$ and $\varphi_{\tau}(\tilde{p})=p$.

We now define the metric $\bar{g}$ as follows. If $X, Y \in T_{p} M$, then

$$
r g(X, Y)=\tilde{g}_{\tau}\left(\left(\left.\left(\varphi_{\tau}\right)_{*}\right|_{\tilde{p}}\right)^{-1}(X),\left(\left.\left(\varphi_{\tau}\right)_{*}\right|_{\tilde{p}}\right)^{-1}(Y)\right)
$$

To show that this metric is well-defined, let $\tilde{p}^{\prime}$ be another such point, i.e. for some $\tau^{\prime}, B_{g_{\tau^{\prime}}}\left(\tilde{p}^{\prime}, r_{1}\right) \subset \mathbb{B}(0, r)$ and $\varphi_{\tau^{\prime}}\left(\tilde{p}^{\prime}\right)=p$. Let $r_{4}=\frac{r}{20} e^{-4 Q}$ and $r_{3}=\frac{r}{20} e^{-3 Q}$. Let $g_{E}$ denote the Euclidean metric. We insert a lemma.

Lemma 3.2. There is an isometric embedding

$$
\psi:\left(\mathbb{B}\left(\tilde{p}, r_{4}\right), g_{\tau}\right) \rightarrow\left(B_{g_{\tau^{\prime}}}\left(\tilde{p}^{\prime}, r_{3}\right), g_{\tau^{\prime}}\right)
$$

Proof. Note that $\varphi_{\tau}$ 's are not diffeomorphisms, hence the lemma is nontrivial.

First $\psi$ can be defined as follows. Since $g_{\tau}$ is $e^{Q}$-quasi-isometric to $g_{E}$,

$$
\mathbb{B}\left(\tilde{p}, r_{4}\right) \subset B_{g_{\tau}}\left(\tilde{p}, r_{3}\right)
$$

For any point $q \in \mathbb{B}\left(\tilde{p}, r_{4}\right)$, connect $q$ to the center point $\tilde{p}$ with a curve $\tilde{\gamma}$ in $\mathbb{B}\left(\tilde{p}, r_{4}\right)$ such that the length of $\tilde{\gamma} l_{g_{\tau}}(\tilde{\gamma})<r_{3}$. Since

$$
\varphi_{\tau}:\left(B_{g_{\tau}}\left(\tilde{p}, r_{3}\right), g_{\tau}\right) \rightarrow B_{g}\left(p, r_{3}\right) \subset M
$$

is a local isometry and $\varphi_{\tau}(\tilde{p})=p$. From $(3.1) \varphi_{\tau}$ maps the curve $\tilde{\gamma}$ to a curve $\gamma$ in $B_{g}\left(p, r_{3}\right)$ starting with $p$ and $l(\gamma)<r_{3}$. Again since $\varphi_{\tau^{\prime}}$ is a local isometry and $\varphi_{\tau^{\prime}}\left(\tilde{p}^{\prime}\right)=p$. The curve $\gamma$ then can be lifted via $\varphi_{\tau^{\prime}}$ to a curve in $B_{g_{\tau^{\prime}}}\left(\tilde{p}^{\prime}, r_{3}\right)$ starting with $\tilde{p}^{\prime}$. The other end point of this curve is defined to be the image of $q$. (Note that, in general, lifting can not be done for incomplete space. Here the map is a local isometry and the curve starts from the center, and we have control on the length of the curve and the size of the metric ball, so it will not hit the boundary during lifting.) Now we will show that $\psi$ is well-defined, i.e. the image is independent of the choices of the curve $\tilde{\gamma}$. If $\tilde{\gamma}_{1}$ is another curve in $\mathbb{B}\left(\tilde{p}, r_{4}\right)$ connecting $q$ to the center point $\tilde{p}$ with $l_{g_{\tau}}\left(\tilde{\gamma}_{1}\right)<r_{3}$, we can connect $\tilde{\gamma}_{1}$ to $\tilde{\gamma}$ by a homotopy $\tilde{H}(s, t)$ in $B_{g_{E}}\left(\tilde{p}, r_{4}\right)$ with fixed end points and $l_{g_{\tau}}(\tilde{H}(s, \cdot))<2 r_{3}$ for each $s$, since $\mathbb{B}\left(\tilde{p}, r_{4}\right)$ is an Euclidean ball and $g_{\tau}$ is $e^{Q_{-}}$quasi-isometric to $g_{E}$. Then $\varphi_{\tau}$ maps $\tilde{H}(s, t)$ to a homotopy $H(s, t)$ in $B_{g}\left(p, 2 r_{3}\right)$ with $l(H(s, \cdot))<2 r_{3}$ for each $s$. Therefore $H(s, t)$ can be lifted via $\varphi_{\tau^{\prime}}$ to a homotopy in $B_{g_{\tau^{\prime}}}\left(\tilde{p}^{\prime}, 2 r_{3}\right)$ starting with $\tilde{p}^{\prime}$. By the (localized) homotopy lifting lemma the other end points are all the same. Therefore $\psi$ is well-defined.

Next we show that $\psi$ is one-to-one. Let $r_{2}=\frac{r}{20} e^{-2 Q}$. Then

$$
B_{g_{\tau^{\prime}}}\left(\tilde{p}^{\prime}, r_{3}\right) \subset \mathbb{B}\left(\tilde{p}^{\prime}, r_{2}\right) \subset B_{g_{\tau^{\prime}}}\left(\tilde{p}^{\prime}, \frac{1}{2} r_{1}\right)
$$


Since $\mathbb{B}\left(\tilde{p}^{\prime}, r_{2}\right)$ is an Euclidean ball one can construct "inverse" $\phi$ similarly as above:

$$
\phi:\left(B_{g_{\tau^{\prime}}}\left(\tilde{p}^{\prime}, r_{3}\right), g_{\tau^{\prime}}\right) \rightarrow\left(B_{g_{\tau}}\left(\tilde{p}, \frac{1}{2} r_{1}\right), g_{\tau}\right) .
$$

Thus $\psi$ is one-to-one. That $\psi$ is an isometric embedding follows from the construction.

Now we continue with the proof of Proposition 3.1. Using the equivariance, we have

$$
\psi^{*} \tilde{g}_{\tau^{\prime}}=\tilde{g}_{\tau} .
$$

Therefore

$$
\begin{aligned}
& \tilde{g}_{\tau^{\prime}}\left(\left(\left.\left(\varphi_{\tau^{\prime}}\right)_{*}\right|_{\tilde{p}^{\prime}}\right)^{-1}(X),\left(\left.\left(\varphi_{\tau^{\prime}}\right)_{*}\right|_{\tilde{p}^{\prime}}\right)^{-1}(Y)\right) \\
& =\tilde{g}_{\tau^{\prime}}\left((\psi)_{*} \circ\left(\left.\left(\varphi_{\tau}\right)_{*}\right|_{\tilde{p}}\right)^{-1}(X),(\psi)_{*} \circ\left(\left(\varphi_{\tau}\right)_{*} \mid \tilde{p}\right)^{-1}(Y)\right) \\
& =\psi^{*} \tilde{g}_{\tau^{\prime}}\left(\left(\left(\varphi_{\tau}\right)_{*} \mid \tilde{p}\right)^{-1}(X),\left(\left(\varphi_{\tau}\right)_{*} \mid \tilde{p}\right)^{-1}(Y)\right) \\
& =\tilde{g}_{\tau}\left(\left(\left(\varphi_{\tau}\right)_{*} \mid \tilde{p}\right)^{-1}(X),\left(\left(\varphi_{\tau}\right)_{*} \mid \tilde{p}\right)^{-1}(Y)\right) .
\end{aligned}
$$

We have proved that the metric $\bar{g}$ is well-defined.

To show that $\varphi_{\tau}^{*} \bar{g}=\tilde{g}_{\tau}$, consider

$$
\varphi_{\tau}: \mathbb{B}\left(0, \frac{9}{10} r\right) \rightarrow U_{\tau}
$$

In particular, for any $\tilde{p} \in \mathbb{B}\left(0, \frac{9}{10} r\right), B_{g_{\tau}}\left(\tilde{p}, r_{1}\right) \subset \mathbb{B}(0, r)$, and therefore $\tilde{p}$ can be used to define the metric $\bar{g}$ at $\varphi_{\tau}(\tilde{p})$. It follows from the definition that

$$
\varphi_{\tau}^{*} \bar{g}=\tilde{g}_{\tau}
$$

Finally, note that the smoothness of the metric $\bar{g}$ is an immediate consequence of (3.2). This completes the proof of Proposition 3.1.

\section{Embedding I}

We continue with the above manifold $(M, g) \in \mathcal{M}(n, \alpha, Q)$. Let $\Omega=\mathbb{B}(0, r) \subset \mathbb{R}^{n}$. We consider the pull back metric $\varphi_{\tau}^{*} g$ on $\Omega$ for a fixed $\tau$. For convenience, this metric will be denoted by $g$. It is easy to see that $\|(\Omega, g)\|_{C^{0, \alpha}, r}^{h} \leq Q(r)$. We are going to construct an equivariant embedding of $(\Omega, g)$ into $L^{2}(\Omega, g)$ by associating to every point $p \in \Omega$ a geometric function $f_{p} \in L^{2}(\Omega) \equiv L^{2}(\Omega, g)$, which depends nicely on $p$. A natural choice seems to be the distance function measured from $p$. Indeed, it is used by Abresch in [A]. However, under our rather weak assumptions 
on the metric it is impossible to have uniform control of the second order derivative of the distance function, which is needed to ensure that the pull-back metric induced by the embedding satisfies a sectional curvature bound. In fact, one can not even expect differentiability of the distance function in balls of uniform size. Our substitute for the distance function is solutions of a canonical geometric partial differential equation. Those solution functions have the crucial equivariance property (like the distance functions) and enjoy better regularity. Many choices of "canonical" PDE solutions are possible, e.g. in [A] Green's function is suggested. But Green's function is inconvenient because of its singularity. We shall employ a very simple and nicely-behaved PDE.

Denote

$$
\Omega_{1}=\Omega \backslash \cup_{q \in \partial \Omega} \overline{B_{g}\left(q, i_{0}\right)},
$$

where $i_{0}=\frac{r}{10}$. $\left(B_{g}(q, \cdot)\right.$ denotes the closed geodesic ball of center $q$ and radius - measured in $g$.) Then for $s \in \Omega_{1}$, let $h_{s} \in L_{0}^{1,2}\left(B_{g}\left(s, i_{0}\right)\right)$ be the unique weak solution of the following Dirichlet boundary value problem:

$$
\begin{cases}\Delta h_{s}=-1 & \text { in } B_{g}\left(s, i_{0}\right) \\ h_{s} \equiv 0 & \text { on } \partial B_{g}\left(s, i_{0}\right)\end{cases}
$$

Here the Laplace operator is defined with respect to the metric $g$. The function $h_{s}$ will be extended to be zero outside the geodesic ball.

First note the following

Lemma 4.1. For all $i_{0} \leq 1$ the following Poincaré inequality holds

$$
\int_{B_{g}\left(s, i_{0}\right)} h^{2} d v \leq C i_{0}^{2} \int_{B_{g}\left(s, i_{0}\right)}|\nabla h|^{2} d v
$$

where $h \in L_{0}^{1,2}\left(B_{g}\left(s, i_{0}\right)\right), d v$ and $\nabla$ are defined with respect to the metric $g$, and $C$ is a uniform constant.

Proof. Since the harmonic $C^{0, \alpha}$-norm of $(\Omega, g)$ is uniformly bounded, we have $B_{g}\left(s, i_{0}\right) \subset \mathbb{B}\left(s, e^{Q(1)} i_{0}\right)$. Now extend $h$ to $\mathbb{B}\left(s, e^{Q(1)} i_{0}\right)$ by defining its value to be zero outside the geodesic ball $B_{g}\left(s, i_{0}\right)$. Then we have

$$
\int_{\mathbb{B}\left(s, e^{Q(1)} i_{0}\right)} h^{2} d v_{E} \leq C e^{2 Q(1)} i_{0}^{2} \int_{\mathbb{B}\left(s, e^{Q(1)} i_{0}\right)}\left|\nabla_{E} h\right|^{2} d v_{E},
$$

where $d v_{E}$ and $\nabla_{E}$ are defined with respect to the Euclidean metric $g_{E}$, and $C$ is a uniform constant. Again since the harmonic $C^{0, \alpha}$-norm of $(\Omega, g)$ is uniformly bounded, the volume element $d v$ and the gradient $\nabla$ are comparable to the Euclidean ones. Therefore the inequality (4.2) follows with a new uniform constant C. 
Using (4.2) and a simple integration argument we then deduce a uniform estimate for the $L^{1,2}$-norm of $h_{s}$ in terms of the volume of $B_{g}\left(s, i_{0}\right)$, namely

$$
\begin{gathered}
\int_{B_{g}\left(s, i_{0}\right)}\left|\nabla h_{s}\right|^{2} \leq C i_{0}^{2} \operatorname{vol}\left(B_{g}\left(s, i_{0}\right)\right), \\
\int_{B_{g}\left(s, i_{0}\right)} h_{s}^{2} \leq C i_{0}^{4} \operatorname{vol}\left(B_{g}\left(s, i_{0}\right)\right) .
\end{gathered}
$$

Here again $C$ is a uniform constant.

Since in harmonic coordinates the Laplace operator takes the form $\Delta=g^{i j} \partial_{i} \partial_{j}$, uniform interior $C^{2, \alpha}$ estimates then follow readily by the standard elliptic theory [GT, Chapter 5]. We also have a uniform $L^{\infty}$ estimate up to boundary, but it seems impossible to obtain better estimate up to boundary because the control of the geometry of the boundary is very weak. At a first glance this appears to threaten to destroy the embedding scheme. Fortunately we can use a cut-off function $\beta=\beta_{n}$ (see (4.9) below) to get around it. On the other hand, we can not obtain control of the dependence of $h_{s}$ on the center $s$. To remedy this, we shall take a suitable average of $h_{s}$ over $s$. The resulting new family of functions will depend nicely on the center.

Now let us state a few more basic properties of the functions $h_{s}$ in the following proposition, which will be proved at the end of this section. Here, as before, we work under the assumption $\|(\Omega, g)\|_{C^{0, \alpha}, r}^{h} \leq Q(r)$.

Proposition 4.2. Let $\bar{h}_{s}(p)$ be the solution of equation (4.1) with respect to the canonical Euclidean metric $g_{E}$ on the Euclidean ball $\mathbb{B}\left(s, i_{0}\right)$. Then for any $\epsilon>0$ and fixed $0<R<1$, there is an $r_{0}=r_{0}(\epsilon, R, Q)>0$ such that if $i_{0} \leq r_{0}$,

$$
\begin{aligned}
\left|h_{s}(p)-\bar{h}_{s}(p)\right| & <\epsilon i_{0}^{2}, \\
\left|\frac{\partial}{\partial p} h_{s}(p)-\frac{\partial}{\partial p} \bar{h}_{s}(p)\right| & <\epsilon i_{0}
\end{aligned}
$$

for all $s$ and all $p$ with $d_{g_{E}}(s, p) \leq R i_{0}$. It will follow from the proof that $\mathbb{B}\left(s, R i_{0}\right) \subset B_{g}\left(s, i_{0}\right)$ so that these estimate make sense. Also

$$
\left|\frac{\partial^{2}}{\partial p^{2}} h_{s}(p)\right| \leq C(n, Q, R), \quad\left|\frac{1}{i_{0}} \frac{\partial}{\partial p} h_{s}(p)\right| \leq C(n, Q, R) .
$$

Remark. Note that

$$
\bar{h}_{s}(p)=\frac{1}{2 n}\left(i_{0}^{2}-d_{g_{E}}^{2}(s, p)\right)
$$

Therefore $\frac{2 n}{i_{0}^{2}} \bar{h}_{s}(p) \leq \frac{1}{5}$ when $d_{g_{E}}(s, p) \geq \sqrt{\frac{4}{5}} i_{0}$. Choosing $R=\frac{10}{11}$ in Proposition 4.2 , we have $\frac{2 n}{i_{0}^{2}} h_{s}(p)<\frac{1}{4}$ when $\sqrt{\frac{4}{5}} i_{0} \leq d_{g_{E}}(s, p) \leq \frac{10}{11} i_{0}$ and $i_{0}$ is sufficiently small. 
Now we proceed to construct the desired embedding. Let $\beta=\beta_{n} \in C_{0}^{\infty}([0, \infty))$ be the cut off function:

$$
\beta_{n}(t)= \begin{cases}0 & \text { if } 0 \leq t \leq \frac{1}{4} \\ B_{n} & \text { if } t \geq \frac{1}{2}\end{cases}
$$

where $B_{n}$ is a constant which will be determined later.

Then $\beta\left(\frac{2 n}{i_{0}^{2}} h_{s}(\cdot)\right)=0$ near the sphere $\partial B_{g}\left(s, \frac{9}{10} i_{0}\right)$ for all $i_{0}$ small. (Note that $d_{g}$ converges to $d_{g_{E}}$ when $i_{0} \rightarrow 0$.) We define a new function which is $\beta\left(\frac{2 n}{i_{0}^{2}} h_{s}(\cdot)\right)$ restricted to the ball $B_{g}\left(s, \frac{9}{10} i_{0}\right)$ and identically zero outside. For simplicity we still denote this new function by $\beta\left(\frac{2 n}{i_{0}^{2}} h_{s}(\cdot)\right)$. As mentioned before, we have no control of the dependence of $h_{s}$ on the center $s$. The said average function is given as follows

$$
f_{p}(q)=\int_{\Omega} \beta\left(\frac{2 n}{i_{0}^{2}} h_{s}(p)\right) \beta\left(\frac{2 n}{i_{0}^{2}} h_{s}(q)\right) d s,
$$

where $d s$ is the volume form of $g$. Note that $f_{p}(q)$ is symmetric in $p$ and $q$ and is $C^{2, \alpha}$ uniformly bounded in both variables.

Now we define the embedding

$$
\begin{aligned}
F: \Omega_{1} & \rightarrow L^{2}(\Omega, g) \\
p & \longmapsto i_{0}^{-\frac{3}{2} n+1} f_{p}
\end{aligned}
$$

Note that

$$
\begin{aligned}
d_{v_{p}} F(q) & =2 n i_{0}^{-\frac{3}{2} n} \int_{\Omega} \beta^{\prime}\left(\frac{2 n}{i_{0}^{2}} h_{s}(p)\right)\left\langle\frac{1}{i_{0}} \nabla h_{s}(p), v_{p}\right\rangle \beta\left(\frac{2 n}{i_{0}^{2}} h_{s}(q)\right) d s \\
\nabla_{v_{p}, w_{p}}^{2} F(q) & =4 n^{2} i_{0}^{-\frac{3}{2} n-1} \\
& \int_{\Omega} \beta^{\prime \prime}\left(\frac{2 n}{i_{0}^{2}} h_{s}(p)\right)\left\langle\frac{1}{i_{0}} \nabla h_{s}(p), v_{p}\right\rangle\left\langle\frac{1}{i_{0}} \nabla h_{s}(p), w_{p}\right\rangle \beta\left(\frac{2 n}{i_{0}^{2}} h_{s}(q)\right) d s \\
& +2 n i_{0}^{-\frac{3}{2} n-1} \int_{\Omega} \beta^{\prime}\left(\frac{2 n}{i_{0}^{2}} h_{s}(p)\right) \nabla^{2} h_{s}(p)\left(v_{p}, w_{p}\right) \beta\left(\frac{2 n}{i_{0}^{2}} h_{s}(q)\right) d s
\end{aligned}
$$

where $v_{p}, w_{p} \in T_{p} \Omega_{1}$.

We first show that when $g$ is the Euclidean metric, we can normalize $\beta$ so that $F$ is an isometric embedding. In this case the embedding function is

$$
\bar{f}_{p}(q)=\int_{\Omega} \beta\left(1-\frac{d_{g_{E}}^{2}(p, s)}{i_{0}^{2}}\right) \beta\left(1-\frac{d_{g_{E}}^{2}(q, s)}{i_{0}^{2}}\right) d s
$$


By the symmetry of the integration domain, $B\left(p, \frac{\sqrt{3} i_{0}}{2}\right) \cap B\left(q, \frac{\sqrt{3} i_{0}}{2}\right)$, and the integrand, $\bar{f}_{p}(q)$ depends only on $d_{g_{E}}(p, q)$ and $B_{n}$. Hence we can write $\bar{f}_{p}(q)=$ $i_{0}^{n} \tilde{f}\left(\frac{1}{i_{0}} d_{g_{E}}(p, q)\right), d_{v_{p}} \bar{F}(q)=i_{0}^{-n / 2} \tilde{f}^{\prime}\left(\frac{1}{i_{0}} d_{g_{E}}(p, q)\right)\left\langle\nabla d_{g_{E}}(p, q), v_{p}\right\rangle$ for a uniquely determined function $\tilde{f}$. Then

$$
\begin{aligned}
\left\|d_{v_{p}} \bar{F}\right\|_{L^{2}(\Omega)}^{2} & =i_{0}^{-n} \int_{B\left(p, 2 i_{0}\right)} \tilde{f}^{\prime 2}\left(\frac{1}{i_{0}} d_{g_{E}}(p, q)\right)\left\langle\nabla d_{g_{E}}(p, q), v_{p}\right\rangle^{2} d q \\
& =i_{0}^{-n} \int_{0}^{2 i_{0}} r^{n-1} \int_{S^{n-1}} \tilde{f}^{\prime 2}\left(\frac{1}{i_{0}} r\right)\left\langle\xi, v_{p}\right\rangle^{2} d \xi d r \\
& =i_{0}^{-n} \frac{\operatorname{vol}\left(S^{n-1}\right)\left|v_{p}\right|^{2}}{n} \int_{0}^{2 i_{0}} r^{n-1} \tilde{f}^{\prime 2}\left(\frac{1}{i_{0}} r\right) d r \\
& =\frac{\operatorname{vol}\left(S^{n-1}\right)\left|v_{p}\right|^{2}}{n} \int_{0}^{2} r^{n-1} \tilde{f}^{\prime 2}(r) d r
\end{aligned}
$$

where $\left|v_{p}\right|$ denote the Euclidean norm of $v_{p}$. Choose $B_{n}$ in the definition of $\beta$ so that $\frac{\operatorname{vol}\left(S^{n-1}\right)}{n} \int_{0}^{2} r^{n-1} \tilde{f}^{\prime 2}(r) d r=1$. Then we arrive at the following.

Lemma 4.3. $\bar{F}$ is an isometric embedding.

With the above choice of $\beta$ we will show that $F$ is an almost isometric embedding when $g$ is not necessarily the Euclidean metric and the second derivative of $F$ is also uniformly bounded. More precisely we have

Proposition 4.4. For any given $\epsilon_{0}>0$, there exists an $r_{0}>0$ such that

$$
\left(1+\epsilon_{0}\right)^{-2} g\left(v_{p}, v_{p}\right) \leq\left\|d_{v_{p}} F\right\|_{L^{2}(\Omega)}^{2} \leq\left(1+\epsilon_{0}\right)^{2} g\left(v_{p}, v_{p}\right),
$$

for all $v_{p} \in T_{p} \Omega_{1}$ and $0<i_{0} \leq r_{0}$. And

$$
\left\|\nabla^{2} F\left(v_{p}, w_{p}\right)\right\|_{L^{2}(\Omega)}^{2} \leq C(n, \alpha, Q) i_{0}^{-2} g\left(v_{p}, v_{p}\right) \cdot g\left(w_{p}, w_{p}\right) .
$$

Proof. By definition

$$
\left\|d_{v_{p}} F\right\|_{L^{2}(\Omega)}^{2}=\int_{B_{g}\left(p, 2 i_{0}\right)}\left|d_{v_{p}} F(q)\right|^{2} d q
$$

To prove (4.13), first note that $\beta, \beta^{\prime}, \beta^{\prime \prime}$ (by construction) and $i_{0}^{-1}\left|\nabla h_{s}\right|$ (by (4.7)) are uniformly bounded. Hence the formulas (4.5) and (4.6) imply (by the theorem of bounded variations) that the difference between the integrand of the formula (4.11) and its Euclidean analog is bounded by $C \epsilon$, where $C$ is a constant independent of $i_{0}$ and the geometry of $(M, g)$. 
On the other hand, the volume element of the metric $g$ satisfies the following inequalities.

$$
e^{-n Q\left(i_{0}\right)} \operatorname{vol}_{R^{n}} \leq \operatorname{vol}_{g} \leq e^{n Q\left(i_{0}\right)} \operatorname{vol}_{R^{n}},
$$

where $Q\left(i_{0}\right) \rightarrow 0$ when $i_{0} \rightarrow 0$. Therefore we obtain an estimate for the difference between $\left\|d_{v_{p}} F\right\|_{L^{2}(\Omega)}^{2}$ and its Euclidean analog which equals $g_{E}(v, v)$. Note that the computation of this difference involves a double integration (one with square), which produces a factor $i_{0}^{3 n}$, killing the factor $i_{0}^{-3 n}$ in the expression (4.11) of $\left|d_{v_{p}} F\right|^{2}$.

The proof of (4.14) is similar. Namely since $\beta, \beta^{\prime}, \beta^{\prime \prime}, i_{0}^{-1}\left|\nabla h_{s}\right|$ and $\left|\nabla^{2} h_{s}\right|$ (by (4.7)) are uniformly bounded by a constant independent of $i_{0}$, so are the integrands of the formula (4.12). Now applying (4.15) and a double integration as above gives the estimate.

Proof of Proposition 4.2. First consider the map

$$
\begin{aligned}
\phi: \mathbb{R}^{n} & \rightarrow \mathbb{R}^{n} \\
\phi(s+x) & =s+i_{0} x .
\end{aligned}
$$

Let $\tilde{h}_{s}(p)$ be the solution of the following problem

$$
\begin{cases}\tilde{\Delta} \tilde{h}_{s}=-1 & \text { in } \phi^{-1}\left(B_{g}\left(s, i_{0}\right)\right) \\ \tilde{h}_{s} \equiv 0 & \text { on } \partial \phi^{-1}\left(B_{g}\left(s, i_{0}\right)\right)\end{cases}
$$

where $\tilde{\Delta}$ is the Laplace operator with respect to the metric $i_{0}^{-2} \phi^{*} g$. Let $\overline{\tilde{h}}_{s}(p)$ denote the solution of the same problem with respect to the Euclidean metric on the Euclidean ball $\mathbb{B}(s, 1)$. Then

$$
\begin{array}{ll}
\overline{\tilde{h}}_{s}(p)=i_{0}^{-2} \bar{h}_{s} \circ \phi(p), & p \in \mathbb{B}(s, 1) \\
\tilde{h}_{s}(p)=i_{0}^{-2} h_{s} \circ \phi(p), & p \in \phi^{-1}\left(B_{g}\left(s, i_{0}\right)\right) .
\end{array}
$$

Since

$$
\left\|\left(\phi^{-1}\left(B_{g}\left(s, i_{0}\right)\right), i_{0}^{-2} \phi^{*} g\right)\right\|_{C^{0, \alpha}, 1}=\left\|\left(B_{g}\left(s, i_{0}\right), g\right)\right\|_{C^{0, \alpha}, i_{0}} \leq Q\left(i_{0}\right)
$$

with $Q\left(i_{0}\right) \rightarrow 0$ as $i_{0} \rightarrow 0$, we have $\left(\phi^{-1}\left(B_{g}\left(s, i_{0}\right)\right), i_{0}^{-2} \phi^{*} g\right) \rightarrow\left(\mathbb{B}(s, 1), g_{E}\right)$ as $i_{0} \rightarrow 0$.

From (4.17) and the estimates for $h_{s}$ (see (4.3), (4.4)) we deduce the following estimates

$$
\int_{\phi^{-1}\left(B_{g}\left(s, i_{0}\right)\right)}\left(\tilde{h}_{s}^{2}+\left|\tilde{\nabla} \tilde{h}_{s}\right|^{2}\right) \leq C(n, Q(1)) .
$$

By the elliptic theory, we then have the following uniform interior estimate:

$$
\left\|\tilde{h}_{s}\right\|_{C^{2, \alpha}\left(B_{i_{0}^{-2} \phi^{*} g}(s, R)\right)} \leq C(n, Q(1), R)
$$


for any fixed $0<R<1$.

From these estimates and the uniqueness of the weak solution $\overline{\tilde{h}}_{s}$ it is easy to deduce the following: for each sequence of centers $s_{k}$ converging to some center $s_{0}$ and each sequence $i_{0}(k)$ converging to zero, the corresponding rescaled solutions $\tilde{h}_{s_{k}}$ converge weakly to $\overline{\tilde{h}}_{s_{0}}$. Moreover, by the Arzela-Ascoli theorem, they also converge uniformly in $C^{1}$ on proper compact subsets of $B_{g_{E}}\left(s_{0}, 1\right)$. This convergence fact along with the smooth dependence of $\overline{\tilde{h}}_{s}$ on $s$ then imply that the $\tilde{h}_{s}$ converge uniformly with respect to $s$ in $C^{1}$ on proper compact subsets of $\mathbb{B}(s, 1)$ as $i_{0}$ goes to zero. Consequently, for a fixed $R \in(0,1)$, given any $\epsilon>0$, there is an $r_{0}>0$ such that for all $s$ and $p$ with $d_{g_{E}}(p, s)<R$, if $i_{0} \leq r_{0}$, then

$$
\left|\tilde{h}_{s}(p)-\overline{\tilde{h}}_{s}(p)\right|<\epsilon \text {. }
$$

Similarly,

$$
\left|\frac{\partial}{\partial p} \tilde{h}_{s}(p)-\frac{\partial}{\partial p} \overline{\tilde{h}}_{s}(p)\right|<\epsilon .
$$

Hence for all $s$ and all $p$ with $d_{g_{E}}(s, p) \leq R i_{0}$,

$$
\begin{aligned}
\left|h_{s}(p)-\bar{h}_{s}(p)\right| & <\epsilon i_{0}^{2}, \\
\left|\frac{\partial}{\partial p} h_{s}(p)-\frac{\partial}{\partial p} \bar{h}_{s}(p)\right| & <\epsilon i_{0} .
\end{aligned}
$$

(4.7) just follows from (4.20) and (4.17).

\section{Embedding II}

In this section we study the geometry of $F\left(\Omega_{1}\right)$ as a submanifold in $L^{2}(\Omega)$. We will prove, among other things, two important properties of $F\left(\Omega_{1}\right)$. That is, the induced metric of $F\left(\Omega_{1}\right)$ has uniformly bounded sectional curvature and the embedding $F$ is equivariant.

The geometry of $F\left(\Omega_{1}\right)$ is completely determined by the second fundamental form of its embedding into $L^{2}(\Omega)$, which in turn can be described by the family of orthogonal projections. $P(y): L^{2}(\Omega) \rightarrow T_{y} F\left(\Omega_{1}\right) \subset L^{2}(\Omega), y \in F\left(\Omega^{1}\right)$. We have

Lemma 5.1. The sectional curvature of $F\left(\Omega_{1}\right)$ is given by the following formula:

$$
R\left(z_{1}, z_{2}\right) z_{3}=\left[d_{z_{1}} P, d_{z_{2}} P\right] z_{3}, \quad z_{1}, z_{2}, z_{3} \in T_{z} F\left(\Omega_{1}\right) .
$$

Proof. Since $P^{2}=P$, one has

$$
\left(d_{z_{1}} P\right) P+P\left(d_{z_{1}} P\right)=d_{z_{1}} P .
$$


Let $\nabla$ be the connection on $F\left(\Omega_{1}\right)$ and $d_{z_{1}}$ the directional derivative on the $L^{2}$ space. Then

$$
\begin{aligned}
\nabla_{z_{1}} z_{2} & =P\left(d_{z_{1}} z_{2}\right) \\
& =d_{z_{1}} z_{2}-(1-P)\left(d_{z_{1}}\left(P z_{2}\right)\right) \\
& =d_{z_{1}} z_{2}-(1-P)\left[\left(d_{z_{1}} P\right) z_{2}+P\left(d_{z_{1}} z_{2}\right)\right] \\
& =d_{z_{1}} z_{2}-(1-P)\left(d_{z_{1}} P\right)\left(P z_{2}\right) \\
& =d_{z_{1}} z_{2}-\left(d_{z_{1}} P\right) z_{2} .
\end{aligned}
$$

Here we have used (5.2) in the last equation. Therefore

$$
\nabla_{z_{1}}=d_{z_{1}}-\left(d_{z_{1}} P\right) .
$$

Now formula (5.1) follows from (5.3) and the definition of the curvature tensor.

Proposition 5.2. Let $\alpha_{0}=\left(1+\epsilon_{0}\right)^{2} C(n, \alpha, Q)$. Here $\epsilon_{0}, r_{0}, C$ are the same constants as in Proposition 4.4. Then for all $0<i_{0}<r_{0}$,

$$
\left\|d_{\dot{y}} P\right\|_{o p} \leq \alpha_{0} i_{0}^{-1}\|\dot{y}\|
$$

Proof. Since $(1-P(F(p))) d_{w_{p}} F=0$,

$$
d_{d_{v_{p}} F} P \cdot d_{w_{p}} F=(1-P(F(p))) \nabla_{v_{p}, w_{p}}^{2} F .
$$

By (4.13) and (4/14), $\left\|d_{\dot{y}} P\right\|_{o p} \leq \alpha_{0} i_{0}^{-1}\|\dot{y}\|$.

Therefore the metric $\tilde{g}=F^{*} g_{L^{2}}$, the metric on $\Omega_{1}$ obtained by pulling back the $L^{2}$ metric, has bounded sectional curvatures.

To prove the equivariance, we first note:

Lemma 5.3. Let $h_{s}(p)$ be the function defined in (4.1), and let $\psi: \Omega \rightarrow \Omega^{\prime}$ be an isometric embedding. Then

$$
h_{\psi(s)}(\psi(p))=h_{s}(p)
$$

Proof. Since equation (4.1) is invariant under isometry, this follows from the uniqueness of solutions to (4.1).

Let $(\Omega, g)$ be as before and $F: \Omega_{1} \rightarrow L^{2}(\Omega)$ the embedding defined in $\S 4$. With the above lemma, we can now prove

Proposition 5.4. If $\psi:(\Omega, g) \rightarrow\left(\Omega^{\prime}, g^{\prime}\right)$ is an isometric embedding, then

$$
\psi:(\Omega, \tilde{g}) \rightarrow\left(\Omega^{\prime}, \tilde{g}^{\prime}\right)
$$


is also an isometric embedding.

Proof. First, we assume $\psi$ is actually an isometry. Then

$$
F \circ \psi(p)=i_{0}^{-\frac{3}{2} n+1} f_{\psi(p)},
$$

where the function

$$
\begin{aligned}
f_{\psi(p)}(q) & =\int_{\Omega}^{\prime} \beta\left(\frac{2 n}{i_{0}^{2}} h_{s}(\psi(p))\right) \beta\left(\frac{2 n}{i_{0}^{2}} h_{s}(q)\right) d s . \\
& =\int_{\Omega} \beta\left(\frac{2 n}{i_{0}^{2}} h_{\psi^{-1}(s)}(p)\right) \beta\left(\frac{2 n}{i_{0}^{2}} h_{\psi^{-1}(s)}\left(\psi^{-1}(q)\right)\right) d s .
\end{aligned}
$$

Here we have used Lemma 5.3. Since $\psi$ is an isometry, a change of coordinates yields

$$
f_{\psi(p)}(q)=f_{p}\left(\psi^{-1}(q)\right)
$$

It follows then that

$$
F \circ \psi=\left(\psi^{-1}\right)^{*} \circ F,
$$

where we have denoted by $\left(\psi^{-1}\right)^{*}$ the map on $L^{2}(\Omega)$ induced by $\psi^{-1}$. Therefore

$$
\psi^{*} F^{*} g_{L^{2}}=(F \circ \psi)^{*} g_{L^{2}}=F^{*}\left(\left(\psi^{-1}\right)^{*}\right)^{*} g_{L^{2}}=F^{*} g_{L^{2}} .
$$

This proves the equivariance when $\psi$ is an isometry. Since $\Omega, \Omega^{\prime}$ are both domains of $R^{n}$, the general statement follows by applying the above to $\psi: \Omega \rightarrow \psi(\Omega)$.

Proof of Theorem 1.1. This theorem is a consequence of Proposition 3.1, Lemma 5.1, Proposition 5.2, Proposition 4.3 and using results from $[\mathrm{A}]$ or $[\mathrm{S}]$, which can be stated that if the sectional curvature is uniformly bounded, then one can smooth the metric so that the weak $C^{k, \alpha}$-norms are uniformly bounded.

\section{Acknowledgment}

We would like to thank the referee for very carefully reading through the paper and many constructive suggestions.

\section{References}

[A] U. Abresch, Über das glatten Riemann'scher metriken, Habilitationsshrift, Rheinischen Friedrich-Wilhelms-Universitat Bonn (1988).

[An] M. Anderson, Convergence and rigidity of manifolds under Ricci curvature bounds, Invent. Math. 102 (1990), 429-445. 
[AC] M. Anderson, J. Cheeger, $C^{\alpha}$-compactness for manifolds with Ricci curvature and injectivity radius bounded below. J. Diff. Geom. 35 (1992), 265-281.

[BMR] J. Bemelmans, Min-Oo, E. Ruh, Smoothing Riemannian metrics, Math. Z. 188 (1984), 69-74.

[CG] J. Cheeger, M. Gromov, On characteristic numbers of complete manifolds of bounded curvature and finite volume, In: Differential Geometry and Complex Analysis, H.E. Rauch Memorial Volume, Springer, New York 1985.

[DWY] X. Dai, G. Wei, R. Ye, Smoothing Riemannian metrics with Ricci curvature bounds, Manu. Math. 90 (1996), 49-61.

[Ga1] Z. Gao, Convergence of Riemannian manifolds, Ricci pinching, and $L^{n / 2}$-curvature pinching, J. Diff. Geom. 32 (1990), 349-382.

[Ga2] Z. Gao, $L^{n / 2}$-curvature pinching, J. Diff. Geom. 32 (1990), 713-774.

[G1] M. Gromov, Almost flat manifolds, J. Diff. Geom. 13 (1978), 231-241.

[G2] M. Gromov, Curvature, diameter and betti numbers, Com. Math. Helv. 56 (1981), 179195.

[H] R.S. Hamilton, Three-manifolds with positive Ricci curvature, J. Diff. Geom. 17 (1982), 255-306.

[JK] J. Jost, H, Karcher, Geometrische methoder zur Gewinnung von a-priori-Schanker für Harmonische Abbildunger, Mann. Math. 40 (1982), 27-77.

[GT] D. Gilbarg, N. Trudinger, Elliptic partial differential equations of second order, 2nd ed., Springer Verlag, 1983.

[P] P. Petersen, Convergence theorems in Riemannian geometry, Comparison Geometry MSRI publications vol 30, 1996.

[PW] P. Petersen, G. Wei, in preparation.

[R] X. Rong, Bounding homotopy and homology groups by curvature and diameter, Duke Math J. 78 (1995), 427-435.

[Sh] Z. Shen, Deforming metrics with curvature and injectivity radius bounded below, Arch. Math. 62 (1994), 354-367.

[S] W.-X. Shi, Deforming the metric on complete Riemannian manifolds, J. Diff. Geom. 30 (1989), 225-301.

[W] Wei, G. Ricci curvature and betti numbers. to appear in J. Geom. Anal..

[Y1] D. Yang, $L^{P}$ pinching and compactness theorems for compact Riemannian manifolds, Séminaire de théorie spectral et géometrie, CHAMBÉRY-GRENOBLE 1987-1988, 81-89.

[Y2] D. Yang, Convergence of Riemannian manifolds with integral bounds on curvature I, Ann. Scient. Ec. Norm. Sup. 25 (1992), 77-105.

Peter Petersen

Department of Mathematics

UCLA

Los Angeles, CA 90095

USA

e-mail: petersen@math.ucla.edu
Guofang Wei and Rugang Ye

Department of Mathematics

University of California

Santa Barbara, CA 93106

USA

e-mail: wei@math.ucsb.edu,yer@math.ucsb.edu

(Received: August 31, 1995) 